\title{
Design of Platform for Service Optimization in Cloud System Environment
}

\author{
Young-Gee Min, Eon-Gon Kim
}

\begin{abstract}
One of the major characteristics of our society in the field of information and communication is its efforts to cope with the rapidly changing IT environment. This is probably because firms are closely related to the survival of the company. As a solution to this problem, researches on cloud computing services are being actively conducted. As cloud computing services have emerged in the marketplace, concepts related to IT assets are changing from being owned to using services. Reflecting this trend, many related companies are providing cloud services. Therefore, in this paper, we design an optimized platform for efficient application of server in cloud system environment. To do this, we designed a system to study various system connections based on the cloud computing service environment and implement an optimized service platform. In this paper, we have implemented optimization and virtualization research in a cloud system environment to design a platform for optimized cloud services. In addition, we designed the optimal platform by studying methodologies such as optimization service environment implementation, compatibility and analysis.
\end{abstract}

Keywords : Cloud system, Cloud service, Cloud server environment, Service optimization, System modeling.

\section{INTRODUCTION}

In the era of the Fourth Industrial Revolution, importance has been emphasized as one of the key technologies, and cloud computing service is emerging as a social requirement. Among them, computer environment is expected to change due to smart system, which is one of cloud service systems based on mobile communication. This makes it easier for users to access content, and anyone can access content easily and conveniently regardless of time or place.

Cloud computing is a computing technology that provides IT resources as a service. It is a technology that provides data center resources to a large number of users in various forms of services based on Internet technology. The cloud computing service is a service using various communication means, and the use and service purchase is not restricted anytime and anywhere. In addition, expansion of services such as infrastructure, platform, and software can be easily and flexibly applied only by the demand of the user, which has a great effect on cost reduction.

In the sharing and management of cloud computing resources, users do not know precisely the location of physical and virtual allocated resources as a security

\section{Revised Manuscript Received on July 22, 2019.}

Young-Gee Min, Ph.D. candidate, Graduate School of Information \& Communications Engineering, Hanbat National University, Korea. Email: jerom72@naver.com

Eon-Gon Kim*, Department of Information \& Communications, Hanbat National University, Korea. Email: egk8996@hanbat.ac.kr characteristic, and operators monitor and record the use of resources to provide optimized services[1][2]. In other words, cloud computing must be provided with all of virtualization, automation, backup, snapshots (monitors and records), and components (services).

Cloud computing is a new technology that provides a large number of integrated computing resources through a variety of services, including infrastructure resources, platforms, and software, through virtualization technology. Computing resources in a cloud computing environment are provided by a data center with thousands of servers. Because a large amount of IT resources are integrated, cloud technologies need to be developed and designed by defining mechanisms and new methods and policies for cloud infrastructure management to provide efficient cloud services[3]. Therefore, operators should be able to optimize the cost of accessing resources by predicting and designing the performance of the services provided by the actual deployment environment and the profit of the provider before distributing the cloud service. In particular, it must be able to coordinate bottlenecks with cloud service requests and offerings. Therefore, it is necessary to predict actual service provision through simulation, not theoretical, subjective, or qualitative measurement. In other words, the platform design for providing optimal cloud service is the most important.

Therefore, in this paper, we design an optimized platform for efficient application of server in cloud system environment. To do this, we designed a system to study various system connections based on the cloud computing service environment and implement an optimized service platform.

\section{EASE OF USE}

In cloud computing, service providers put a variety of resources (hardware, platform, and software) related to IT in a huge cloud of web, and users who need resources connect to the web and borrow various services You can think of it as a paid service. In other words, it focuses on shared services and serves the entire resources such as infrastructure, platforms, and applications.

In general, cloud computing can be divided into SaaS, IaaS, and PaaS. Software as a Service (SaaS) is a form of providing a software application as a service. One of the advantages of SaaS service is that you can run the program without installing the required software. 
In addition, it is a form of service that you do not need to purchase a lot of complicated and various software separately and can use it immediately without installation[4].

IaaS (Infrastructure as a Service) is a service that provides server infrastructure as a service. It provides storage or compute through the Internet. It is not only a software service but also a hardware service. This means borrowing storage space or computing power[5].

Platform as a Service (PaaS) is a combination of SaaS and IaaS, which provides an environment in which users can develop software. It includes compilation language, web programs, and production tools. You can create and use a new application. Figure 1 illustrates the concept of cloud computing services[6].

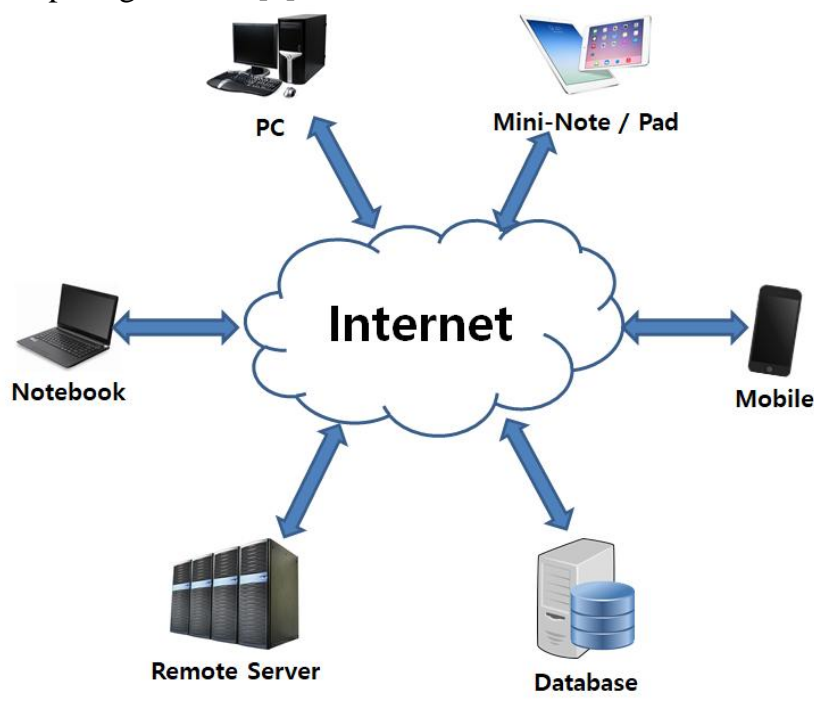

Fig 1 .Cloud computing concept

In recent years, cloud computing has required security controls such as access control management, data encryption, distributed management, availability and integrity in a virtualized environment. Virtualization refers to the ability to consolidate or separate physical IT resources into one. Distributed management and availability are the technologies required to manage integrated data resources. Access control management, data encryption and integrity are also required to protect shared IT resources[7][8]. As such, research is under way to eliminate the risks that may arise in cloud computing.

This is because cloud computing is expected to lead to revolutionary changes in the corporate IT environment. Cloud computing has been accepted as a technology that can share IT resources to improve management efficiency, reduce costs, and dramatically improve the ability to use computers. Despite expectations, however, cloud computing has been slow to actual adoption. The reason for this is that the benefits are not enough, but there is a lack of related services and solutions, and companies are burdened with pioneering adoption. However, many industry stakeholders and experts predict that the market, technology and services of cloud computing will become more visible from this year[9]. Now that the conceptual review has been underway, it is estimated that the real environment has been established to take advantage of cloud computing.

So far, the computer environment will evolve from a personal computer environment to a networking computer environment in the future. Therefore, each user connected to the global network will be transformed into an environment that provides vast amount of data and resources. This change has gradually changed the perception that data can be occupied if only the personal computer is occupied until now. Therefore, Google is sharing the Excel word with the web, and then bringing the data back to the web through the network, and editing and storing the data. As the environment becomes more environmentally friendly, the software is borrowed rather than purchased by individuals. It is changing. After all, the better your network infrastructure is, the better you will be able to take advantage of your cloud computing system[10].

Analysts expect hybrid cloud computing to combine the power and control of a private cloud with the cost-effectiveness of a public cloud. Companies are constantly striving to advance into the market ahead of their time by developing more innovative ideas, products and services in a rapidly changing global competitive environment. Cloud computing offers new possibilities to further develop the enterprise and leverage a cost-effective infrastructure.

\section{VIRTUALIZATION DESIGN}

Virtualization is a way to separate computing resources and physical characteristics, such as the interaction of other systems, applications, or end users. Virtualization technology allows users to leverage computing capabilities from one application to the entire operating system without being tied to specific physical hardware or other resources that support it. In other words, by separating the logical areas of physical equipment, computing resources can be freed from existing complex devices to be utilized and managed in the most efficient manner. Virtualization can be combined to dynamically combine computing components, regardless of changes in configuration or new patches, upgrades, or changes in the user or application side, to ensure the best delivery experience[11]. Figure 2 shows a model of virtualization for an optimized environment of cloud services.

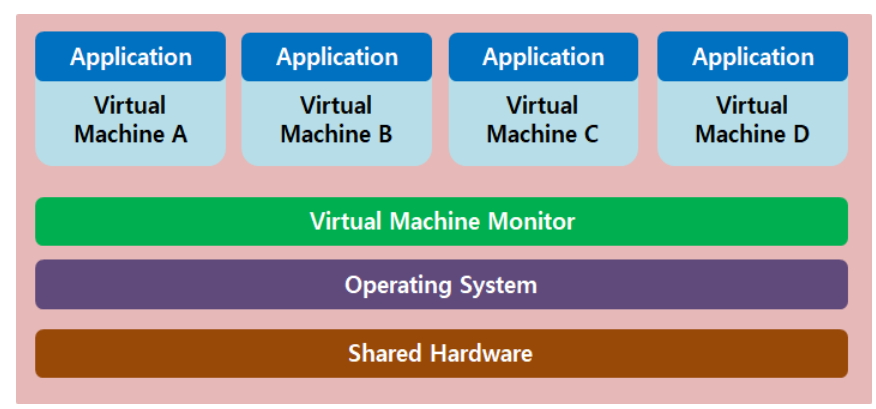

Fig 2. Cloud service virtualization modeling

The service business that leverages the cloud system is under pressure to ease management and reduce costs while maintaining and enhancing competitive advantages such as flexibility, reliability, scalability or security. 
Virtualization will help you consolidate multiple servers in a single system while addressing separate maintenance. One of the key benefits of server consolidation is not only to reduce hardware requirements, but also to achieve significant organizational cost savings through the adoption of networked storage for managing and sharing data in an organization that is increasingly conserving strategic and administrative costs[12].

These cloud virtualizations include server virtualization, application virtualization, and desktop virtualization. Server virtualization has already become a cornerstone for cost reduction and data center optimization, and is increasingly becoming the cornerstone of cloud computing. However, for efficient operation of virtualized servers, it is necessary to have an infrastructure for this, especially a networking environment. With server virtualization technology, you can utilize the system by separating it into virtual units without being bound to physical resources such as CPU or server. For putting different operating systems on top of each other, or using multiple servers as a single server, thereby increasing resource utilization and improving manageability. Figure 3 shows server virtualization and network virtualization.
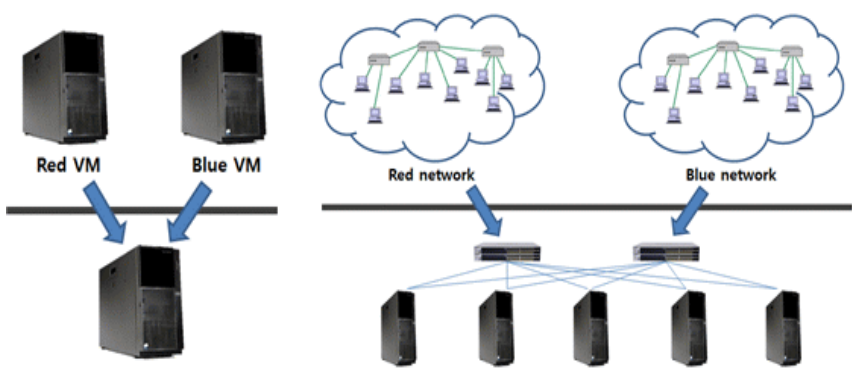

Fig 3. Server \& Network virtualization

Next, application virtualization includes server-side application virtualization (or presentation virtualization) and client-side application virtualization (or application streaming). Application virtualization allows users to use desktop applications without having to install them all when they want to use them in a variety of physical environments such as PCs, laptops, PDAs, or other personal devices. In practice, you install the application on a central server and send only the virtual interface over the network. When the user uses the application, the keyboard or mouse click information is sent back to the server through the network, so that the screen transmits the updated information to the user device so that virtually no data is stored in the user device.

Virtualization redirects isolate the underlying operating system and applications, improving application manageability and compatibility. With application virtualization, applications are not installed in the traditional sense. Instead, the application is packaged in a way that provides each application in an isolated runtime environment that operates using the virtualized instances of the required system services, settings, and data. When a user requests an application, the package is streamed to the user's system. That is, as the sending process proceeds, the application is sent over the network in a manner that is executed. This approach provides access to applications when the user is offline, example, you could divide a server into multiple servers,

providing additional benefits for the organization to fully exploit the end user's computing power.

Finally, server-based desktop virtualization separates the end user's work or interaction from the physical desktop. Users work as local devices, such as PCs or thin clients, but the computing environment in which they interact is actually a remote system, usually a data center server. Only inputs such as the user's keyboard and mouse clicks are transmitted over the network to the remote system, and the user interface, such as the virtual desktop, is presented to the end user again over the network[12].

For IT organizations, desktop management is a time-consuming and costly task. However, desktop virtualization can be easily upgraded from the data center to the latest desktop environment through virtualization technology. It has the advantage of lowering costs in management and faster user support, and users can concentrate on maintaining the latest desktop environment without having to wait for support. Figure 4 shows desktop virtualization.

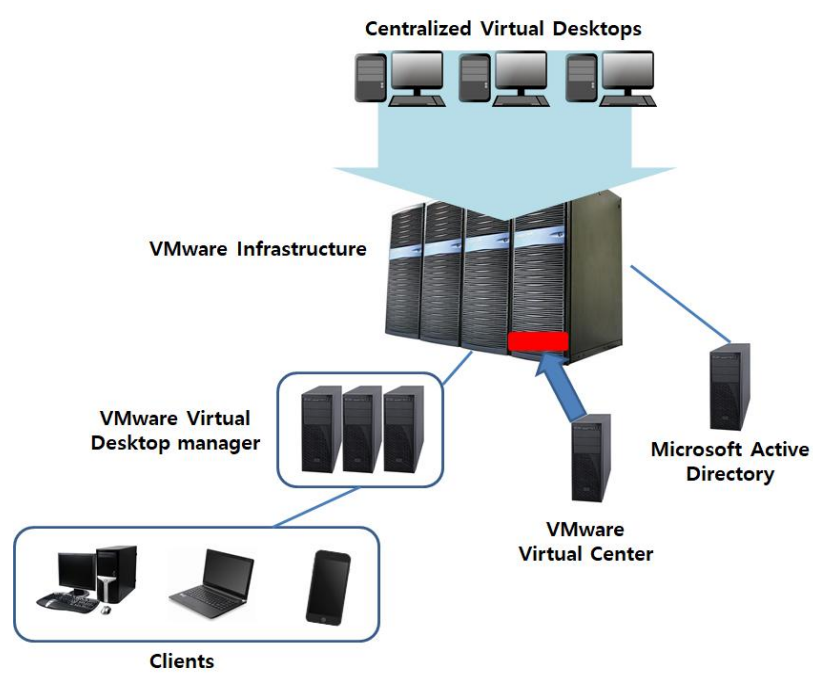

Fig 4. Desktop virtualization

\section{OPTIMIZATION PLATFORM DESIGN}

In this paper, we design an optimized platform for efficient application of server in cloud system environment. To do this, we designed a system to study various system connections based on the cloud computing service environment and implement an optimized service platform.

For the optimization test of cloud service, we modeled service model of cloud computing network environment using Datacenter, Host, Virtual Machine, Cloudlet provided by CloudSim. Since there is a queue that manages the cloudlet in the virtual machine, the system is a virtual machine from the viewpoint of queuing theory. Therefore, Figure 5 shows the $\mathrm{M}$ / M / C model with multiple virtual machines that provide the host resources in the datacenter as a service type.

As shown in Figure 5, the cloud service model has a "work request time" requesting service at T0 and a "waiting time" $\mathrm{T} 1$, which is the time to wait in queue before allocating resources 
if data center resources are insufficient. T0 is implemented to occur as an exponential distribution, which is a distribution requesting work in an actual network, and these requests are generated by random numbers. The service process proceeds in the order in which the tasks are requested and queues if there are no available processing elements (PEs). When the requested service is assigned a resource, the task is executed in T2 and the task is completed in T3. T4 is the time when the user is provided with all the services.

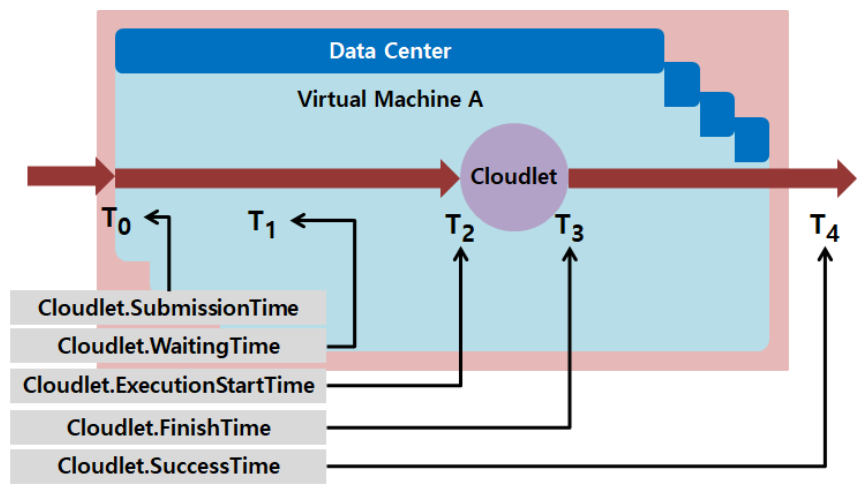

Fig 5. Cloud service model

Based on the above model, you can analyze and modify the predictFileTransferTime () function of the Datacenter class provided by CloudSim, the submitCloudlet () function of the DatacenterBroker class, all the functions of the VM class, and the updateVM Processing () and CloudletSubmit () functions of the SpaceSharedVMScheduler class and outputs the optimization index value for the service.

The average waiting time is the average time that the user's request, which occurs in the random number of the exponential distribution, is queued. E (T4 - T0) is the average time taken by the user to request the service (T0) and to receive the service (T4). The average queue length is the average length of the variable queues that exist in the virtual machine according to the service request. The work throughput is the amount of work the server handles per hour, and the server utilization rate is the total server drive time ratio that handles the user's request for $\mathrm{T} 0$ where exponential distribution occurs with a random number $(\lambda)$ while the server is running.

One operator with a data center holds 10 hosts in one datacenter, and each host has one virtual machine with optimized performance. Datacenter's IT resources are managed by First-Come First-Served (FCFS) policies. Businesses use virtual machines, which are virtualization technologies, to provide IT resources to users. Virtual machines are used by SpaceSharedAllocationVM Split virtual machine allocation). And the Virtual Machine provides users with Cloudlet using SpaceSharedSchedule. As the number of user requests increases, the use of the IT resources of the Datacenter increases because the cloudlet is activated. In this case, the service provider should provide optimized service in terms of availability as the user increases by comparing the number of user requests that the system can accommodate, the available computing resources, the service cost of the user and the service waiting time.

Cloud service cost is the cost that a user requesting a
Cloudlet should pay. In addition, Datacenter Cost is a cost incurred when a virtual machine creates an IT resource within a Datacenter. At present, the cost of Datacenter is constantly charged because there is one datacenter and one operator. However, the total cloud service cost increases proportionally with the demand of the cloudlet. In order for operators to avoid deficits due to the cost of managing virtual machines in Datacenter, cloud service costs should be higher than Datacenter costs. Therefore, at least 20 services must be provided.

\section{CONCLUSION}

One of the major characteristics of our society in the field of information and communication is its efforts to cope with the rapidly changing IT environment. To cope with the diverse business environment, companies are constantly investing in IT-related purchases for storage and servers.

Therefore, in this paper, we design an optimized platform for efficient application of server in cloud system environment. To do this, we designed a system to study various system connections based on the cloud computing service environment and implement an optimized service platform. In this paper, we have implemented optimization and virtualization research in a cloud system environment to design a platform for optimized cloud services. In addition, we designed the optimal platform by studying methodologies such as optimization service environment implementation, compatibility and analysis.

Cloud service was modeled based on queuing theory and CloudSim, which provides simulation of cloud computing environment, was modified to calculate the availability index of network service. Based on this, we used CloudSim simulator to test the availability of cloud services and find a place to optimize. Therefore, developers will be able to determine the appropriate number, performance, and configuration of Datacenter servers to provide cloud services using this methodology before deploying a cloud computing environment. As a result, systems and services can be optimized prior to deploying cloud services, reducing the cost and effort of rebuilding the system

\section{APPENDIX}

It is optional. Appendixes, if needed, appear before the acknowledgment.

\section{ACKNOWLEDGMENT}

It is optional. The preferred spelling of the word "acknowledgment" in American English is without an "e" after the "g." Use the singular heading even if you have many acknowledgments. Avoid expressions such as "One of us (S.B.A.) would like to thank ... ." Instead, write "F. A. Author thanks" Sponsor and financial support acknowledgments are placed in the unnumbered footnote on the first page. 


\section{REFERENCES}

1. A. K. Sidhu, S. Kinger, "Analysis of load balancing techniques in cloud computing," International Journal of Computers and Technology, 4(2), 2013, pp.737-741. DOI : 10.1.1.799.8142\&rep

2. J. M. Bohli, N. Gruschka, M. Jensen, L. L. Iacono, N. Marnau, "Security and Privacy-Enhancing Multi Cloud Architectures," IEEE Transaction on Dependable and Secure Computing, 10(4), 2013, pp.212-224. DOI: 10.1109/TDSC.2013.6

3. B. Hayes, "Cloud computing," Communications of the ACM, 51(7), 2008, pp.9-11. DOI : 10.1145/1364782.1364786

4. R. Rajeshkannan, M. Aramudhan, "Comparative study of Load Balancing Algorithms in cloud computing environment," Indian Journal of Science and Technology, 9(20), 2016, pp.1-7. DOI: 10.1109/ICECDS.2017.8389549

5. N. Yager, A. Amin, "Fingerprint verification based on minutiae features: a review," Pattern Anal Appl., 7(1), 2004, pp.94-113. https://doi.org/10.1007/s10044-003-0201-2

6. H. Shen, G. Liu, "An efficient and trustworthy resource sharing platform for collaborative cloud computing," IEEE Transactions on Parallel and Distributed Systems, 25(4), 2014, pp.862-875

7. M. U. Bokhari, M. Alam, F. Hasan, "Performance analysis of dynamic load balancing algorithm for multiprocessor interconnection network," Perspectives in Science (PICS), 8, 2016, pp.564-566. https://doi.org/10.1016/j.pisc.2016.06.021

8. I. A. Rassan, H. A. Shaher, "Securing Mobile Cloud Using Finger Print Authentication," International Journal of Network Security \& Its Applications (IJNSA), 5(6), 2013, pp.41-53.

9. C. Zhu, H. Nicanfar, V. C. Leung, L. T. Yang, "An authenticated trust and reputation calculation and management system for cloud and sensor networks integration," IEEE Transactions on Information Forensics and Security, 10(1), 2015, pp.118-131.

10. M. Li, "An approach to reliably identifying signs of DDOS flood attacks based on LRD traffic pattern recognition," Computer \& Security, 23, 2004, pp.549-558. DOI : 10.1016/j.cose.2004.04.005

11. J. Mirkovic, J. Martin, T. Reiher, "A taxonomy of DDoS attacks and DDoS defense mechanisms," ACM SIGCOMM Computer Communication Review, 34(2), 2004, pp.39-53. DOI : 10.1145/997150.997156

12. F. Cohen, "Simulating cyber attacks, defences, and consequences," Computer \& Security, 18, 1999, pp.479-518.

\section{AUTHORS PROFILE}

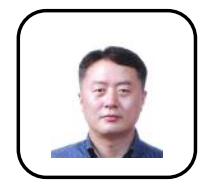

Young-Gee Min Received the M.S degrees from the Department of Computer Engineering in Hanbat National University. $\mathrm{He}$ is currently PM in ABLE information technology. His research interests include Cloud computing, Big data, System \& Network security.

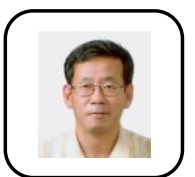

Eon-Gon Kim was born in Busan ,Korea in 1958. He received his B.S and M.S degrees in Aerospace Electronic Engineering from Korea Aerospace University, Korea, in 1985 and 1997 respectively. From 1985 to 1994,he worked as cellular system engineer at Samsung Electronic. From 1995, he is currently a professor in the department of information \& communication engineering. His research interests include mobile communication, WBAN, and wireless modem 